\title{
MICELLAR EFFECT ON METAL-LIGAND COMPLEXES OF Co(II), Ni(II), Cu(II) AND Zn(II) WITH CITRIC ACID
}

\author{
Srinivasa Rao Pedada, Srikanth Bathula, Samba Siva Rao Vasa, Kamala Sastry Charla and \\ Nageswara Rao Gollapalli*
}

School of Chemistry, Andhra University, Visakhapatnam-530 003, India

(Received December 4, 2008; revised August 10, 2009)

\begin{abstract}
Chemical speciation of citric acid complexes of $\mathrm{Co}(\mathrm{II}), \mathrm{Ni}(\mathrm{II}), \mathrm{Cu}$ (II) and $\mathrm{Zn}$ (II) was investigated $\mathrm{pH}$-metrically in $0.0-2.5 \%$ anionic, cationic and neutral micellar media. The primary alkalimetric data were pruned with SCPHD program. The existence of different binary species was established from modeling studies using the computer program MINIQUAD75. Alkalimetric titrations were carried out in different relative concentrations (M:L:X = 1:2:5, 1:3:5, 1:5:3) of metal $(\mathrm{M})$ to citric acid. The selection of best chemical models was based on statistical parameters and residual analysis. The species detected were $\mathrm{MLH}, \mathrm{ML}_{2}, \mathrm{ML}_{2} \mathrm{H}$ and $\mathrm{ML}_{2} \mathrm{H}_{2}$. The trend in variation of stability constants with change in mole fraction of the medium is explained on the basis of electrostatic and non-electrostatic forces. Distributions of the species with $\mathrm{pH}$ at different compositions of micellar media are also presented.
\end{abstract}

KEY WORDS: Chemical speciation, Citric acid, Micelles, Metals, Binary complexes

\section{INTRODUCTION}

Speciation study of essential metal ion complexes is useful to understand the role played by the active site cavities in biological molecules and the bonding behavior of protein residues with the metal ion. Cobalt acts as catalyst in a variety of enzyme system functions and as coenzymes in several biochemical processes. Cobalt is essential for the production of red blood cells. In this respect its roles range from weak ionic enzymatic cofactors to highly specific substances known as metalloenzymes [1]. Nickel is an essential nutrient. It is also a component of the enzyme, urease, present in a wide range of plant species [2]. Copper is an essential element for life and one of the transition elements frequently found at the active sites of proteins. Copper proteins are essential constituents of aerobic organisms, including hemocyanins and enzymes that activate $\mathrm{O}_{2}$, promoting oxygen atom incorporation into biological substrates. Zinc is an essential trace metal after iron and it plays important roles in biological systems [3-6]. Zinc is associated with both the exchangeable and non-exchangeable pools in human plasma.

Citric acid is the most common and abundant means of strong respiratory energy in fruits besides carbohydrates [7] and represents an easily metabolisable source of energy in mammals. It acts as an anti-rachitic agent in primates [8]. Infection of the prostate gland is accompanied by a decrease in the concentration of citric acid in case of Chlamydia trachomatis infection of prostate [9]. Citric acid acts as a strong iron chelator to prevent and reverse the formation of ferric cyanide-3-glucoside complexes in dislocation of skin [10]. Ni(II)-citric acid complex inhibited glucose metabolism by the bacteria. It is also used in the synthesis of cerium oxide [11] and zinc oxide [12] nanoparticles by different methods. Citric acid is involved in cis-platin incorporated hyaluronic acid nanoparticles based on ion-complex formation [13].

The role of micelles is simple, but vital resulting in compartmentalization of the energy transfer partners. It separates non-polar molecules from each other by encapsulating them in hydrophobic cavity. Additionally the microenvironment created around the micelle favors dissociation of complexes. By binding the metal ions electrostatically to the negatively charged

*Corresponding author. E-mail: raosrp@gmail.com 
micellar surface, their effective concentration is substantially increased. The concentration of these counter cations in the micellar surface has been estimated [14] to be around $3.0 \mathrm{M}$. Literature survey $[15,16]$ shows that increased concentration of micellised surfactant leads to the distribution of the reactants over a large amount of micelles. It leads to a dilution of reactants in the micellar pseudo phase and decreases the observed rate constants. Micellar effects on reactivity are generally independent of changes in micellar size and shape. The effect of micelles on overall reaction rates and equilibria depends upon the incorporation of solutes into the micellar pseudo phase. The second reason is that the species with lower charge or high hydrophobicity are stabilized in the micellar pseudo phase. So metal ligand combinations resulting in neutral complexes will be favored. It has been demonstrated that water insoluble metal chelates can be solubilised with a micellar solution of non-ionic surfactant such as Triton $\mathrm{X}-100$. This solubilisation technique is frequently utilized in analytical procedures [17]. Since Triton $\mathrm{X}-100$ is a non-ionic surfactant, it is expected to destabilize the charged species in aqueous solutions.

In recent years modeling studies involving binary complexes have gained popularity [1821]. Hence, the present study was taken up based on the importance of the chemical speciation of essential metal ions with bio-ligands in micellar medium. This study also assumes importance since the micellar effect on the chemical speciation of citric acid complexes has not been studied so far.

\section{EXPERIMENTAL}

\section{Chemicals and reagents}

Solutions of $\mathrm{Co}(\mathrm{II}), \mathrm{Ni}(\mathrm{II}), \mathrm{Cu}(\mathrm{II})$ and $\mathrm{Zn}$ (II) chlorides and citric acid were prepared by dissolving G.R. Grade (Merck, India) salts in triple distilled water. To increase the solubility of citric acid and to suppress the hydrolysis of metal salts, mineral acid concentration in the above solutions was maintained at $0.05 \mathrm{M}$. AR samples of sodium laurylsulphate (SLS, Qualigens, India), cetyltrimethylammonium bromide (CTAB, Qualigens, India) and Triton X-100 (TX100, E-Merck, Germany) were used as such and their purity was checked by determining critical micellar concentration (CMC) conductometrically. The CMC values of SLS, CTAB and TX100 were $0.0084 \mathrm{M}, 0.00093 \mathrm{M}$ and $0.0089 \mathrm{M}$, respectively, at $303 \mathrm{~K}$. To assess the errors that might have crept into the determination of the concentrations, the data were subjected to analysis of variance of one way classification (ANOVA). The strength of alkali was determined using the Gran plot $[22,23]$ method.

\section{Apparatus}

The titrations were carried out in the medium containing varying concentrations $(0.5-2.5 \%)$ of micellar medium maintaining an ionic strength of $0.16 \mathrm{M}$ with sodium nitrate at $303.0 \pm 0.1 \mathrm{~K}$. An ELICO (Model L1-120, India) pH-meter (readability 0.01) was used to monitor the changes in $\mathrm{H}^{+}$concentration. The $\mathrm{pH}$ meter was calibrated with $0.05 \mathrm{M}$ potassium hydrogen phthalate in acidic region and $0.01 \mathrm{M}$ borax solution in basic region. The glass electrode was equilibrated in a well stirred micellar-water mixture containing inert electrolyte. The effect of variations in asymmetry, liquid junction potential, activity coefficient, sodium ion error and dissolved carbon dioxide on the response of glass electrode were accounted for in the form of correction factor [24]. The emf of the cell may be expressed by the equation $E=E^{0}+(R T / F) \ln a_{H}{ }^{+}$or $E=E^{0}+$ $0.059 \mathrm{pH}$ at $25^{\circ} \mathrm{C}$, where $\mathrm{E}^{0}$ is a constant partly dependent upon the nature of the glass used for making the membrane. The value of $\mathrm{E}^{0}$ may vary slightly with time, and it is related to the existence of an asymmetry potential [25] in a glass electrode. Owing to the asymmetry potential, if a glass electrode is inserted into a test solution, which is identical with the internal $\mathrm{HCl}$ 
Micellar effect on metal-ligand complexes of $\mathrm{Co}(\mathrm{II}), \mathrm{Ni}(\mathrm{II}), \mathrm{Cu}(\mathrm{II})$ and $\mathrm{Zn}$ (II) with citric acid 349

solution, the electrode shall have a small potential which is found to vary with time. Hence, glass electrode was standardized frequently using a buffer of known hydrogen activity.

\section{Procedure}

Titrations of strong acid with alkali were carried out at regular intervals to check whether complete equilibrium was achieved. The calomel electrode was refilled with surfactant-water mixture of equivalent composition as that of the titrand. In each of the titrations, the titrand consisted of 1-3 mmol of mineral acid in a total volume of $50 \mathrm{~mL}$. Titrations with different ratios $(1: 2.5,1: 3.5,1: 5: 3)$ of metal-ligand were carried out with $0.4 \mathrm{M}$ sodium hydroxide. The titrations were carried out in media containing varying amounts of surfactants (SLS, CTAB and TX100) maintaining an ionic strength of $0.16 \mathrm{M}$ with $\mathrm{NaNO}_{3}$ at $303.0 \mathrm{~K}$. In these titrations, the titrand consisted of mineral acid $\left(\mathrm{HNO}_{3}\right)$ and the ligand (citric acid) in the presence of metal ion $(\mathrm{Co}, \mathrm{Ni}, \mathrm{Cu}$ and $\mathrm{Zn}$ ) in a total volume of $50 \mathrm{~mL}$. Titrations were performed by adding each time $0.1 \mathrm{~mL}$ portions of sodium hydroxide (ca., $0.4 \mathrm{M}$ ) to the titrand. The $\mathrm{pH}$ meter dial reading was recorded only after a constant value was displayed [26].

\section{Modeling strategy}

The approximate complex stability constants of citric acid were calculated with the computer program SCPHD [27]. The best fit chemical model for each system investigated was arrived at using MINIQUAD75. The variation of stability constants of metal-ligand species was analyzed by electrostatic grounds and on the basis of solute-solute and solute-solvent interactions.

\section{RESULTS AND DISCUSSION}

Alkalimetric titration curves in surfactant-water mixtures revealed that the acid-base equilibria of citric acid are active in the pH-range 1.75-6.50. Based on the active forms of the ligand in this $\mathrm{pH}$-range, models containing various numbers and combination of complex species were generated using an expert system package CEES [28]. These models were inputted to the computer program, MINIQUAD75 [29] along with the alkalimetric titration data. The best fit model was selected using the statistical parameters of the least square residuals. The final models are given in Tables 1-3 along with the statistical parameters.

\section{Effect of systematic errors on best fit model}

In order to rely upon the best chemical model for critical evaluation and application under varied experimental conditions with different accuracies of data acquisition, an investigation was made by introducing pessimistic errors in the influential parameters like concentrations of alkali, mineral acid, ligand and metal (Table 4). The order of the ingredients that influence the magnitudes of stability constants due to incorporation of errors is alkali $>$ acid $>$ ligand $>$ metal. Some species are even rejected when errors are introduced in the concentrations. The rejection of some species and increased standard deviations in the stability constants on introduction of errors confirm the appropriateness of the experimental conditions (concentrations of ingredients) and the choice of the best fit models. 
Table 1. Parameters of best fit chemical models of $\mathrm{Co}(\mathrm{II}), \mathrm{Ni}(\mathrm{II}), \mathrm{Cu}(\mathrm{II})$ and $\mathrm{Zn}$ (II)-citric acid complexes in CTAB-water mixtures.

\begin{tabular}{|c|c|c|c|c|c|c|c|c|c|c|c|}
\hline \multirow{2}{*}{$\begin{array}{l}\% \mathrm{w} / \mathrm{v} \\
\mathrm{CTAB}\end{array}$} & \multicolumn{4}{|c|}{$\log \beta_{\operatorname{mlh}}(\mathrm{SD})$} & \multirow[t]{2}{*}{$\mathrm{NP}$} & \multirow[t]{2}{*}{$\begin{array}{c}\mathrm{U}_{\text {corr }} \times \\
10^{8}\end{array}$} & \multirow[t]{2}{*}{$\begin{array}{l}\text { Skew } \\
\text {-ness }\end{array}$} & \multirow[t]{2}{*}{$\chi^{2}$} & \multirow[t]{2}{*}{ R-Factor } & \multirow[t]{2}{*}{ Kurtosis } & \multirow[t]{2}{*}{$\mathrm{pH}$ range } \\
\hline & MLH & $\mathrm{ML}_{2}$ & $\mathrm{ML}_{2} \mathrm{H}$ & $\mathrm{ML}_{2} \mathrm{H}_{2}$ & & & & & & & \\
\hline \multicolumn{12}{|c|}{$\mathrm{Co}$ (II) } \\
\hline 0.0 & $9.87(2)$ & $7.78(3)$ & $12.60(4)$ & $17.88(15)$ & 104 & 5.05 & -1.22 & 97.34 & 0.0152 & 8.32 & $2.00-6.50$ \\
\hline 0.5 & $8.37(1)$ & $8.33(6)$ & $12.25(7)$ & $16.08(8)$ & 100 & 1.51 & -1.59 & 100.3 & 0.0162 & 4.37 & $2.00-5.50$ \\
\hline 1.0 & $8.61(2)$ & $7.75(3)$ & $13.17(10)$ & $18.07(12)$ & 98 & 7.02 & -0.69 & 44.31 & 0.0206 & 6.71 & \begin{tabular}{|l|}
$2.00-7.50$ \\
\end{tabular} \\
\hline 1.5 & $8.41(2)$ & $8.09(4)$ & 13.61(4) & $17.30(10)$ & 114 & 7.05 & 0.74 & 38.22 & 0.0084 & 10.54 & \begin{tabular}{|l|}
$1.90-6.00$ \\
\end{tabular} \\
\hline 2.0 & $8.57(3)$ & $8.62(3)$ & $13.57(5)$ & $\begin{array}{l}17.57(6) \\
\end{array}$ & 95 & 3.12 & 1.73 & 45.42 & 0.0742 & 7.92 & $2.50-7.00$ \\
\hline 2.5 & $9.98(1)$ & $9.21(3)$ & $15.02(7)$ & $18.32(8)$ & 107 & 7.30 & -0.03 & 29.65 & 0.0041 & 1.91 & $2.00-6.50$ \\
\hline \multicolumn{12}{|c|}{$\mathrm{Ni}(\mathrm{II})$} \\
\hline 0.0 & $9.98(2)$ & $8.97(5)$ & $14.17(4)$ & $17.85(8)$ & 72 & 3.83 & 0.12 & 98.34 & 0.0061 & 3.17 & $2.00-5.00$ \\
\hline 0.5 & $9.23(2)$ & $7.21(3)$ & $13.05(4)$ & $17.10(8)$ & 97 & 1.89 & 1.24 & 101.3 & 0.0031 & 11.12 & $1.65-6.50$ \\
\hline 1.0 & $9.54(3)$ & $7.48(4)$ & $13.47(5)$ & $17.32(7)$ & 95 & 1.57 & 0.15 & 92.30 & 0.0053 & 10.03 & $2.30-6.70$ \\
\hline 1.5 & $9.69(2)$ & $7.67(3)$ & 13.64(4) & $17.45(6)$ & 93 & 4.14 & 0.58 & 48.46 & 0.0154 & 7.54 & $2.00-6.90$ \\
\hline 2.0 & $9.84(1)$ & $7.76(2)$ & $13.72(5)$ & $17.69(8)$ & 95 & 8.17 & -0.55 & 36.65 & 0.0094 & 6.32 & $2.00-6.50$ \\
\hline 2.5 & $10.02(3)$ & $7.89(3)$ & $13.94(7)$ & $17.88(11)$ & 90 & 3.78 & -0.67 & 23.24 & 0.0742 & 5.05 & $2.00-6.00$ \\
\hline \multicolumn{12}{|c|}{$\mathrm{Cu}(\mathrm{II})$} \\
\hline 0.0 & $94(1)$ & $11.67(4)$ & & $.10(6)$ & 120 & 8.38 & 0.22 & 63.23 & 0.0052 & 4.1 & $2.00-6.00$ \\
\hline 0.5 & $8.43(2)$ & $7.56(3)$ & 14.64(4) & $17.22(7)$ & 125 & 9.83 & 0.72 & 74.72 & 0.0065 & 8.02 & $2.00-5.70$ \\
\hline 1.0 & $8.64(1)$ & $7.63(3)$ & 14.71(5) & $17.45(10)$ & 120 & 0.42 & 0.84 & 59.56 & 0.0894 & 6.32 & $2.00-6.70$ \\
\hline 1.5 & $8.36(1)$ & $7.52(2)$ & $14.49(5)$ & $17.03(8)$ & 111 & 9.90 & 1.01 & 84.02 & 0.0047 & 4.01 & $2.00-6.40$ \\
\hline 2.0 & $8.87(3)$ & $8.11(4)$ & $15.15(4)$ & $17.88(6)$ & 88 & 9.00 & 1.92 & 93.87 & 0.0214 & 2.84 & $2.40-6.30$ \\
\hline 2.5 & $9.25(1)$ & $8.36(2)$ & $15.22(5)$ & $18.08(8)$ & 104 & 3.76 & 2.04 & 45.85 & 0.0075 & 7.32 & $2.50-6.50$ \\
\hline \multicolumn{12}{|c|}{$\mathrm{Zn}(\mathrm{II})$} \\
\hline 0.0 & -- & $7.84(2)$ & $12.82(5)$ & $16.79(8)$ & 127 & 4.79 & -1.97 & 25.42 & 0.0021 & 9.71 & $1.90-6.70$ \\
\hline 0.5 & -- & $6.22(3)$ & $11.25(4)$ & 15.34(8) & 124 & 4.22 & -2.02 & 47.14 & 0.0052 & 3.02 & $1.85-6.50$ \\
\hline 1.0 & -- & $6.34(4)$ & $11.69(5)$ & $15.52(7)$ & 126 & 4.19 & -2.11 & 38.04 & 0.0365 & 5.55 & $1.85-6.70$ \\
\hline 1.5 & -- & $6.55(4)$ & $11.84(2)$ & 15.79(12) & 115 & 0.70 & -2.34 & 36.66 & 0.0074 & 7.01 & $1.95-6.90$ \\
\hline 2.0 & -- & $6.25(2)$ & $11.33(4)$ & $15.28(6)$ & 103 & 5.98 & -1.94 & 47.05 & 0.0095 & 4.08 & $1.75-6.00$ \\
\hline 2.5 & -- & $7.32(3)$ & $12.25(5)$ & $16.30(8)$ & 105 & 2.79 & 0.82 & 65.21 & 0.0415 & 2.11 & $2.00-6.70$ \\
\hline
\end{tabular}

Table 2. Parameters of best fit chemical models of $\mathrm{Co}(\mathrm{II}), \mathrm{Ni}(\mathrm{II}), \mathrm{Cu}(\mathrm{II})$ and $\mathrm{Zn}$ (II)-citric acid complexes in TX100-water mixtures.

\begin{tabular}{|c|c|c|c|c|c|c|c|c|c|c|c|}
\hline \multirow{2}{*}{$\begin{array}{r}\% \mathrm{v} / \mathrm{v} \\
\mathrm{TX} 100\end{array}$} & \multicolumn{4}{|c|}{$\log \beta_{\mathrm{mlh}}(\mathrm{SD})$} & \multirow[t]{2}{*}{$\mathrm{NP}$} & \multirow{2}{*}{$\begin{array}{c}\mathrm{U}_{\text {corr }} \times \\
10^{8} \\
\end{array}$} & \multirow{2}{*}{$\begin{array}{l}\text { Skew } \\
\text {-ness }\end{array}$} & \multirow[t]{2}{*}{$\chi^{2}$} & \multirow[t]{2}{*}{ R-Factor } & \multirow[t]{2}{*}{ Kurtosis } & \multirow[t]{2}{*}{$\mathrm{pH}$ range } \\
\hline & MLH & $\mathrm{ML}_{2}$ & $\mathrm{ML}_{2} \mathrm{H}$ & $\mathrm{ML}_{2} \mathrm{H}_{2}$ & & & & & & & \\
\hline \multicolumn{12}{|c|}{$\mathrm{Co}(\mathrm{II})$} \\
\hline 0.0 & $9.87(2)$ & $7.78(3)$ & $12.60(4)$ & $17.88(15)$ & 104 & 5.05 & -1.22 & 97.34 & 0.0152 & 8.32 & $2.00-6.50$ \\
\hline 0.5 & $8.86(2)$ & $6.89(8)$ & $11.33(9)$ & $17.54(20)$ & 132 & 1.12 & 0.94 & 22.04 & 0.0195 & 7.62 & $1.60-6.20$ \\
\hline 1.0 & $8.48(2)$ & $7.25(7)$ & $12.67(8)$ & $17.63(18)$ & 145 & 1.02 & -0.52 & 76.02 & 0.0083 & 5.38 & $1.75-7.50$ \\
\hline 1.5 & $8.54(4)$ & $7.62(7)$ & $12.83(8)$ & 17.71(18) & 140 & 9.63 & -0.84 & 101.5 & 159 & 3.39 & \begin{tabular}{|l|}
$1.75-7.50$ \\
\end{tabular} \\
\hline 2.0 & $8.80(3)$ & $6.88(6)$ & $12.52(7)$ & $17.52(17)$ & 105 & 6.97 & -0.41 & 99.04 & 0.0140 & 4.78 & $2.15-8.01$ \\
\hline 2.5 & 8.91(3) & 7.81(5) & $13.15(5)$ & $17.25(18)$ & 106 & 4.21 & 0.01 & 82.20 & 0.0011 & 5.59 & $2.00-5.65$ \\
\hline \multicolumn{12}{|c|}{$\mathrm{Ni}(\mathrm{II})$} \\
\hline 0.0 & $9.98(2)$ & $8.61(5)$ & $14.17(4)$ & $17.85(8)$ & 72 & 3.83 & 0.12 & 98.34 & 0.0061 & 3.17 & $2.00-5.00$ \\
\hline 0.5 & $8.65(2)$ & $7.85(8)$ & $12.49(12)$ & $16.25(20)$ & 125 & 1.60 & -1.42 & 35.92 & 0.0532 & 5.54 & $1.85-6.90$ \\
\hline 1.0 & $8.63(3)$ & $7.62(8)$ & 12.94(11) & $17.65(21)$ & 154 & 5.08 & -2.32 & 83.38 & 0.1626 & 4.75 & $1.75-6.00$ \\
\hline 1.5 & $8.43(3)$ & $7.49(7)$ & $12.73(12)$ & $17.74(22)$ & 150 & 2.93 & -1.04 & 64.32 & 0.0153 & 3.32 & \begin{tabular}{|l|}
$1.75-6.20$ \\
\end{tabular} \\
\hline 2.0 & $9.02(2)$ & 7.31(6) & $12.97(11)$ & $17.38(18)$ & 100 & 3.06 & 1.09 & 76.14 & 0.0082 & 6.05 & $2.00-5.50$ \\
\hline 2.5 & $8.69(2)$ & $7.05(6)$ & $12.88(14)$ & 17.39(17) & 98 & 3.89 & 1.11 & 65.46 & 0.0054 & 4.80 & $2.00-5.25$ \\
\hline
\end{tabular}

Bull. Chem. Soc. Ethiop. 2009, 23(3) 
Micellar effect on metal-ligand complexes of $\mathrm{Co}(\mathrm{II}), \mathrm{Ni}(\mathrm{II}), \mathrm{Cu}(\mathrm{II})$ and $\mathrm{Zn}$ (II) with citric acid 351

\begin{tabular}{|c|c|c|c|c|c|c|c|c|c|c|c|}
\hline \multicolumn{10}{|c|}{$\mathrm{Cu}(\mathrm{II})$} \\
\hline 0.0 & $9.94(1)$ & $11.67(4)$ & $16.83(4)$ & $18.10(6)$ & 120 & 8.38 & 0.22 & 63.23 & 0.0052 & 4.14 & $2.00-6.00$ \\
\hline 0.5 & $8.96(2)$ & $8.05(7)$ & $12.81(10)$ & $17.31(21)$ & 115 & 9.46 & -1.74 & 23.81 & 0.0843 & 3.79 & $2.00-5.85$ \\
\hline 1.0 & $8.74(3)$ & $7.55(8)$ & $12.80(12)$ & $17.07(18)$ & 144 & 1.10 & -1.82 & 34.32 & 0.0423 & 5.44 & $1.75-6.00$ \\
\hline 1.5 & $8.98(2)$ & $8.17(8)$ & $14.08(12)$ & $17.96(15)$ & 79 & 7.38 & 0.42 & 79.73 & 0.0382 & 4.84 & $2.00-5.50$ \\
\hline 2.0 & $9.14(3)$ & $8.19(8)$ & $13.33(12)$ & $18.11(19)$ & 117 & 6.14 & 0.01 & 81.89 & 0.0193 & 3.66 & $2.00-6.40$ \\
\hline 2.5 & $9.04(2)$ & $8.07(9)$ & $13.3(13)$ & $17.78(21)$ & 123 & 7.55 & -5.7 & 106.12 & 0.0370 & 4.26 & $2.00-5.75$ \\
\hline \multicolumn{10}{|c|}{ Zn(II) } \\
\hline 0.0 & -- & $7.84(2)$ & $12.82(5)$ & $16.79(8)$ & 127 & 4.79 & -1.97 & 25.42 & 0.0021 & 9.71 & $1.90-6.70$ \\
\hline 0.5 & -- & $5.72(2)$ & $11.62(8)$ & $16.28(13)$ & 130 & 4.85 & 0.55 & 52.84 & 0.0094 & 5.92 & $1.85-6.50$ \\
\hline 1.0 & -- & $7.07(3)$ & $13.02(5)$ & $17.25(14)$ & 149 & 4.24 & 0.04 & 42.45 & 0.0078 & 4.32 & $1.75-7.00$ \\
\hline 1.5 & -- & $7.84(3)$ & $13.84(7)$ & $18.28(13)$ & 150 & 2.93 & -0.21 & 82.32 & 0.0092 & 7.42 & $1.75-7.20$ \\
\hline 2.0 & -- & $5.88(4)$ & $12.63(7)$ & $17.71(14)$ & 86 & 4.54 & 0.01 & 38.31 & 0.0178 & 5.13 & $2.50-6.95$ \\
\hline 2.5 & -- & $6.34(4)$ & $13.15(5)$ & $18.01(15)$ & 80 & 0.25 & 0.13 & 43.13 & 0.0428 & 2.76 & $2.50-7.50$ \\
\hline
\end{tabular}

Table 3. Parameters of best fit chemical models of $\mathrm{Co}(\mathrm{II}), \mathrm{Ni}(\mathrm{II}), \mathrm{Cu}$ (II) and $\mathrm{Zn}$ (II)-citric acid complexes in SLSwater mixtures.

\begin{tabular}{|c|c|c|c|c|c|c|c|c|c|c|c|}
\hline \multirow{2}{*}{$\begin{array}{c}\% \mathrm{w} / \mathrm{v} \\
\text { SLS }\end{array}$} & \multicolumn{4}{|c|}{$\log \beta_{\operatorname{mlh}}(\mathrm{SD})$} & \multirow[t]{2}{*}{$\mathrm{NP}$} & \multirow[t]{2}{*}{$\begin{array}{c}\mathrm{U}_{\text {corr } X} \times \\
10^{8} \\
\end{array}$} & \multirow[t]{2}{*}{$\begin{array}{l}\text { Skew } \\
\text {-ness }\end{array}$} & \multirow[t]{2}{*}{$\chi^{2}$} & \multirow[t]{2}{*}{ R-Factor } & \multirow[t]{2}{*}{ Kurtosis } & \multirow[t]{2}{*}{$\mathrm{pH}$ range } \\
\hline & MLH & $\mathrm{ML}_{2}$ & $\mathrm{ML}_{2} \mathrm{H}$ & $\mathrm{ML}_{2} \mathrm{H}_{2}$ & & & & & & & \\
\hline \multicolumn{12}{|c|}{$\mathrm{Co}(\mathrm{II})$} \\
\hline 0.0 & $9.87(2)$ & $7.78(3)$ & $12.60(4)$ & $17.88(15)$ & 104 & 5.05 & -1.22 & 97.34 & 0.0153 & 8.32 & $2.0-6.50$ \\
\hline 0.5 & $9.73(2)$ & $8.97(9)$ & $13.53(12)$ & $17.94(18)$ & 65 & 6.29 & -0.32 & 25.34 & 0.0043 & 3.32 & $2.5-6.50$ \\
\hline 1.0 & $9.74(3)$ & 8.79(7) & 13.97(11) & $17.59(18)$ & 62 & 3.22 & -0.82 & 32.23 & 0.0814 & 4.71 & $2.5-6.20$ \\
\hline 1.5 & $9.83(4)$ & $8.65(8)$ & 13.75(12) & $17.42(18)$ & 60 & 6.84 & -0.73 & 89.32 & 0.0297 & 2.21 & $2.5-6.50$ \\
\hline 2.0 & $9.59(4)$ & $8.54(7)$ & $13.66(13)$ & $17.29(15)$ & 78 & 2.94 & -0.52 & 26.63 & 0.0018 & 4.16 & $2.5-7.00$ \\
\hline 2.5 & $9.00(3)$ & $8.34(7)$ & $13.47(15)$ & $17.14(19)$ & 74 & 7.89 & -1.42 & 73.60 & 0.0062 & 3.81 & $2.5-6.90$ \\
\hline \multicolumn{12}{|c|}{$\mathrm{Ni}(\mathrm{II})$} \\
\hline 0.0 & $9.98(2)$ & $8.97(5)$ & $14.17(4)$ & $17.85(8)$ & 72 & 3.83 & 0.12 & 98.34 & 0.0061 & 3.17 & $2.0-5.00$ \\
\hline 0.5 & $9.54(2)$ & $8.54(6)$ & $13.75(7)$ & $17.82(20)$ & 57 & 6.85 & -1.45 & 21.42 & 0.0321 & 2.32 & $2.5-6.50$ \\
\hline 1.0 & $9.32(3)$ & $8.43(6)$ & $13.43(9)$ & $17.59(21)$ & 59 & 0.17 & -2.24 & 29.92 & 0.0480 & 4.32 & $2.5-6.50$ \\
\hline 1.5 & $9.07(3)$ & $8.11(5)$ & $13.53(10)$ & $17.43(22)$ & 62 & 4.03 & -0.75 & 35.47 & 0.0057 & 4.82 & $2.0-6.50$ \\
\hline 2.0 & $9.51(4)$ & $8.03(7)$ & $13.70(10)$ & $16.97(23)$ & 76 & 7.80 & -0.36 & 42.37 & 0.0299 & 5.57 & $2.0-7.50$ \\
\hline 2.5 & $9.83(4)$ & $8.28(7)$ & 13.82(11) & $16.84(23)$ & 64 & 3.68 & -1.98 & 32.32 & 0.0020 & 8.6 & $2.0-6.30$ \\
\hline \multicolumn{12}{|c|}{$\mathrm{Cu}(\mathrm{II})$} \\
\hline 0.0 & $9.94(1)$ & $11.67(5)$ & $16.83(4)$ & $18.10(6)$ & 120 & 8.38 & 0.22 & 63.23 & 0.0052 & 4.14 & $2.0-6.00$ \\
\hline 0.5 & $10.69(2)$ & $8.65(5)$ & $15.68(7)$ & $18.79(18)$ & 58 & 2.00 & -2.14 & 15.21 & 0.0284 & 5.41 & $2.5-6.50$ \\
\hline 1.0 & $10.85(3)$ & $8.78(6)$ & $15.74(8)$ & $18.57(18)$ & 61 & 0.34 & -2.45 & 18.42 & 0.0573 & 4.24 & $2.5-6.00$ \\
\hline 1.5 & $10.93(4)$ & $8.69(7)$ & $15.58(8)$ & $18.47(17)$ & 64 & 8.64 & -4.72 & 20.32 & 0.0487 & 8.60 & $2.5-6.50$ \\
\hline 2.0 & $10.51(2)$ & 8.03(7) & $15.72(7)$ & $18.23(18)$ & 76 & 7.80 & -2.04 & 25.04 & 0.0299 & 2.49 & $2.0-7.50$ \\
\hline 2.5 & $10.03(2)$ & $7.95(8)$ & $15.05(8)$ & $18.15(17)$ & 60 & 3.68 & -3.72 & 11.91 & 0.1960 & 5.54 & $2.0-6.50$ \\
\hline \multicolumn{12}{|c|}{$\mathrm{Zn}(\mathrm{II})$} \\
\hline 0.0 & -- & $7.84(2)$ & $12.82(5)$ & $16.79(8)$ & 127 & 4.79 & -1.97 & 25.42 & 0.0021 & 9.71 & $1.9-6.70$ \\
\hline 0.5 & -- & $8.21(3)$ & $15.18(15)$ & $16.95(21)$ & 68 & 7.07 & 0.32 & 26.88 & 0.0749 & 3.42 & $2.5-6.50$ \\
\hline 1.0 & -- & $8.11(2)$ & 15.14(15) & $16.72(22)$ & 71 & 6.76 & 1.29 & 30.75 & 0.0487 & 5.41 & $2.5-7.00$ \\
\hline 1.5 & -- & $8.01(2)$ & $15.12(16)$ & 16.51(21) & 79 & 5.71 & 0.20 & 49.93 & 0.0446 & 2.36 & $2.0-7.50$ \\
\hline 2.0 & -- & $8.64(3)$ & $15.23(16)$ & $16.29(22)$ & 70 & 6.11 & 1.18 & 82.80 & 0.0035 & 3.88 & $2.5-7.00$ \\
\hline 2.5 & -- & $8.85(4)$ & $15.15(15)$ & $16.12(20)$ & 68 & 4.92 & 1.25 & 29.83 & 0.0025 & 4.16 & $2.5-6.50$ \\
\hline
\end{tabular}


Table 4. Effect of errors in influential parameters on the stability constants of $\mathrm{Zn}$ (II) citric acid complexes in $0.5 \% \mathrm{w} / \mathrm{v}$ CTAB-water mixture.

\begin{tabular}{|l|c|l|l|l|}
\hline \multirow{3}{*}{ Ingredient } & \multirow{3}{*}{ \% Error } & \multicolumn{3}{|c|}{$\log \beta(\mathrm{SD})$} \\
\cline { 2 - 5 } & \multicolumn{4}{|c|}{ Citric acid } \\
\hline \multirow{4}{*}{ Alkali } & 0 & $6.72(5)$ & $11.75(4)$ & $15.64(4)$ \\
\cline { 2 - 5 } & -5 & $7.09(15)$ & Rejected & Rejected \\
\cline { 2 - 5 } & -2 & Rejected & $11.94(41)$ & Rejected \\
\cline { 2 - 5 } & +2 & Rejected & Rejected & $15.25(23)$ \\
\cline { 2 - 5 } Acid & +5 & Rejected & $11.37(32)$ & Rejected \\
\hline \multirow{5}{*}{ Ligand } & -5 & Rejected & $11.41(22)$ & Rejected \\
\cline { 2 - 5 } & -2 & $6.23(21)$ & Rejected & Rejected \\
\cline { 2 - 5 } & +2 & Rejected & $11.34(30)$ & Rejected \\
\cline { 2 - 5 } & +5 & $6.29(41)$ & Rejected & Rejected \\
\cline { 2 - 5 } & -5 & $6.23(12)$ & $11.31(10)$ & $15.26(90)$ \\
\cline { 2 - 5 } & -2 & $6.21(22)$ & $11.29(10)$ & $15.24(66)$ \\
\cline { 2 - 5 } & +2 & $6.25(49)$ & $11.34(62)$ & $15.31(84)$ \\
\hline \multirow{5}{*}{ Metal } & +5 & $6.31(97)$ & $11.39(14)$ & $15.88(25)$ \\
\cline { 2 - 5 } & -5 & $6.22(38)$ & $11.29(41)$ & $15.24(70)$ \\
\cline { 2 - 5 } & -2 & $6.24(37)$ & $11.21(41)$ & $15.69(70)$ \\
\cline { 2 - 5 } & +2 & $6.70(36)$ & $11.35(42)$ & $15.31(69)$ \\
\cline { 2 - 5 } & +5 & $6.31(35)$ & $11.39(55)$ & $15.33(69)$ \\
\hline
\end{tabular}

\section{Effect of micelles}

Variations in the magnitudes of formation constant of the complex species formed due to the interaction between metal ions and citric acid with mole fraction of the surfactants are shown in Figures 1-3 which indicate that the stabilities of the complexes are increasing linearly with mole fraction of the CTAB and decreasing linearly with SLS, TX100 media. The decreased stability of the complex with increased surfactant concentration may be due to the increased number of micelles and decreased dielectric constant of the medium. The dielectric constant of the medium has a direct influence on the protonation-deprotonation equilibria [30, 31]. The variation of $\log$ $\mathrm{K}^{\mathrm{M}}$ or change in free energy $\left(\Delta \mathrm{G}^{\mathrm{t}}\right)$ with surfactant content can be written on the sum of the changes in $\Delta \mathrm{G}$ due to electro static and non-electrostatic factors.

$$
\text { i.e. } \quad \begin{aligned}
\Delta G^{t} & =R T\left(\ln K^{M}-\ln K^{W}\right) \\
& =\Delta G_{e l}+\Delta G_{n e l}
\end{aligned}
$$

Born's classical treatment [32] holds good in accounting for the electrostatic contribution to the free energy change. According to this treatment, the energy of electrostatic interaction is related to dielectric constant, $\mathrm{D}_{\mathrm{s}}$ of the medium and is given by

$$
\Delta G_{e l}=\frac{r e^{2}}{z} * \frac{1}{D_{s}} * \frac{1}{r}
$$

Hence, equations 1 and 2 suggest that the logarithm of stability constant should vary linearly as a function of the reciprocal of the dielectric constant of the medium. The Stern layer [33] of the cationic micelles (CTAB) has positively charged head groups and anions shall be present in Gouy-Chapmann double layer. Hence, the positively charged complexes should be stabilized on the surface of the micelles. The species should be stabilized in the micellar medium with opposite charges by CTAB (Figure 1) due to electrostatic interactions. In remaining cases, stability of the binary complexes decreases linearly with increased SLS content (Figure 3). As

Bull. Chem. Soc. Ethiop. 2009, 23(3) 
Micellar effect on metal-ligand complexes of $\mathrm{Co}(\mathrm{II}), \mathrm{Ni}(\mathrm{II}), \mathrm{Cu}(\mathrm{II})$ and $\mathrm{Zn}$ (II) with citric acid 353

TX100 is a neutral surfactant; hence, the stabilities of charged species are decreased (Figure 2) due to the decreased dielectric constant of the medium.

\section{Distribution diagrams}

Citric acid is a tridentate ligand and it has three protonation constants (Table 5). It exists as $\mathrm{LH}_{3}$, $\mathrm{LH}_{2}^{-}, \mathrm{LH}^{2-}$ and $\mathrm{L}^{3-}$ in the $\mathrm{pH}$ regions 1.75-3.0, 2.0-4.5, 3.5-5.5 and 4.0-8.0, respectively (Scheme 1) [34]. The protonation equilibria of L-aspartic acid, citric acid and succinic acid in anionic and cationic micellar media was earlier reported [34, 35].

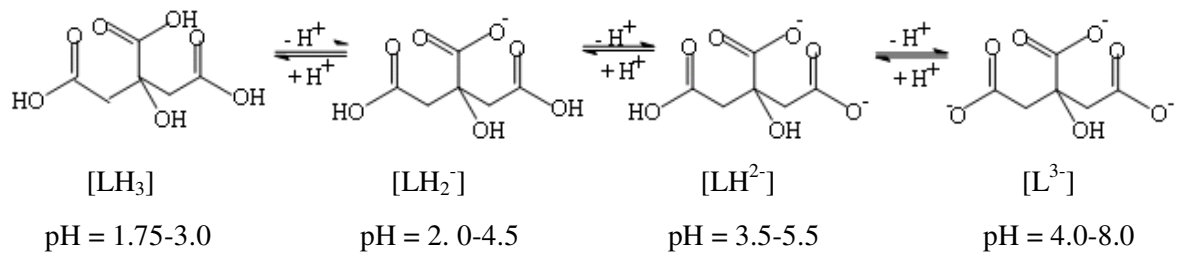

Scheme 1. Protonation-deprotonation equilibria of citric acid.
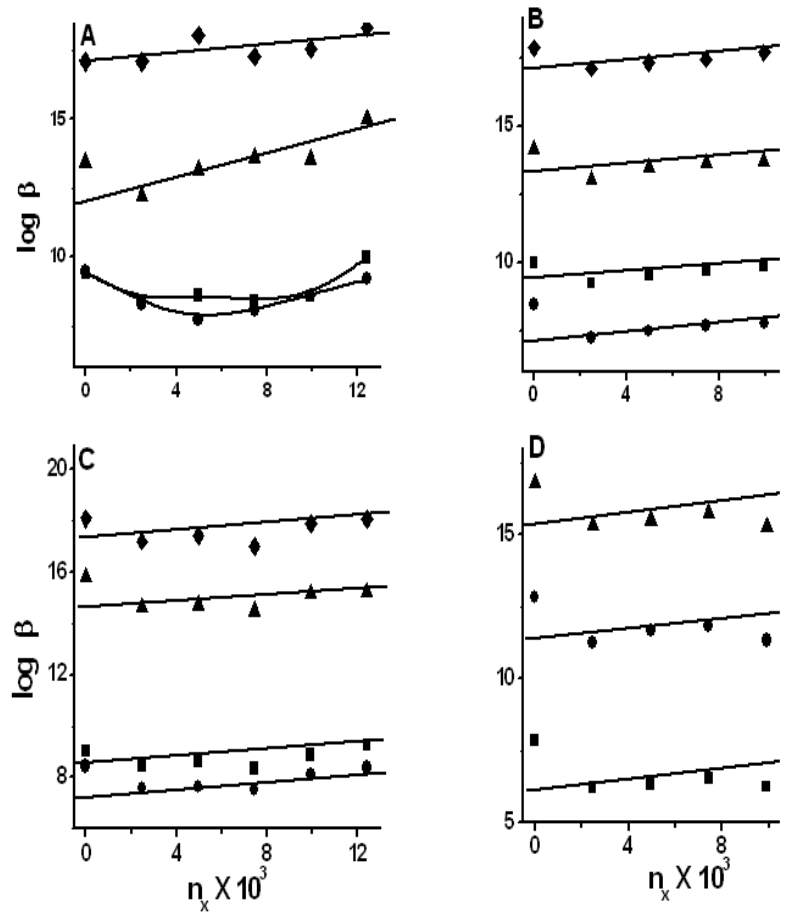

Figure 1. Variation of stability constant values of metal-citric acid complexes with mole fraction

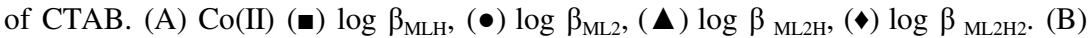
$\mathrm{Ni}(\mathrm{II})(\boldsymbol{\bullet}) \log \beta_{\mathrm{MLH}},(\bullet) \log \beta_{\mathrm{ML} 2},(\boldsymbol{\Delta}) \log \beta_{\mathrm{ML} 2 \mathrm{H}},(\bullet) \log \beta_{\mathrm{ML} 2 \mathrm{H} 2}$. (C) $\mathrm{Cu}$ (II) (অ) $\log \beta_{\mathrm{MLH}}$, $(\bullet) \log \beta_{\mathrm{ML} 2},(\mathbf{\Delta}) \log \beta_{\mathrm{ML} 2 \mathrm{H}},(\bullet) \log \beta_{\mathrm{ML} 2 \mathrm{H} 2}$ (D) $\mathrm{Zn}(\mathrm{II})(\bullet) \log \beta_{\mathrm{ML} 2},(\bullet) \log \beta_{\mathrm{ML} 2 \mathrm{H}}$, ( $\left.\mathbf{\Delta}\right)$ $\log \beta_{\mathrm{ML} 2 \mathrm{H} 2}$. 

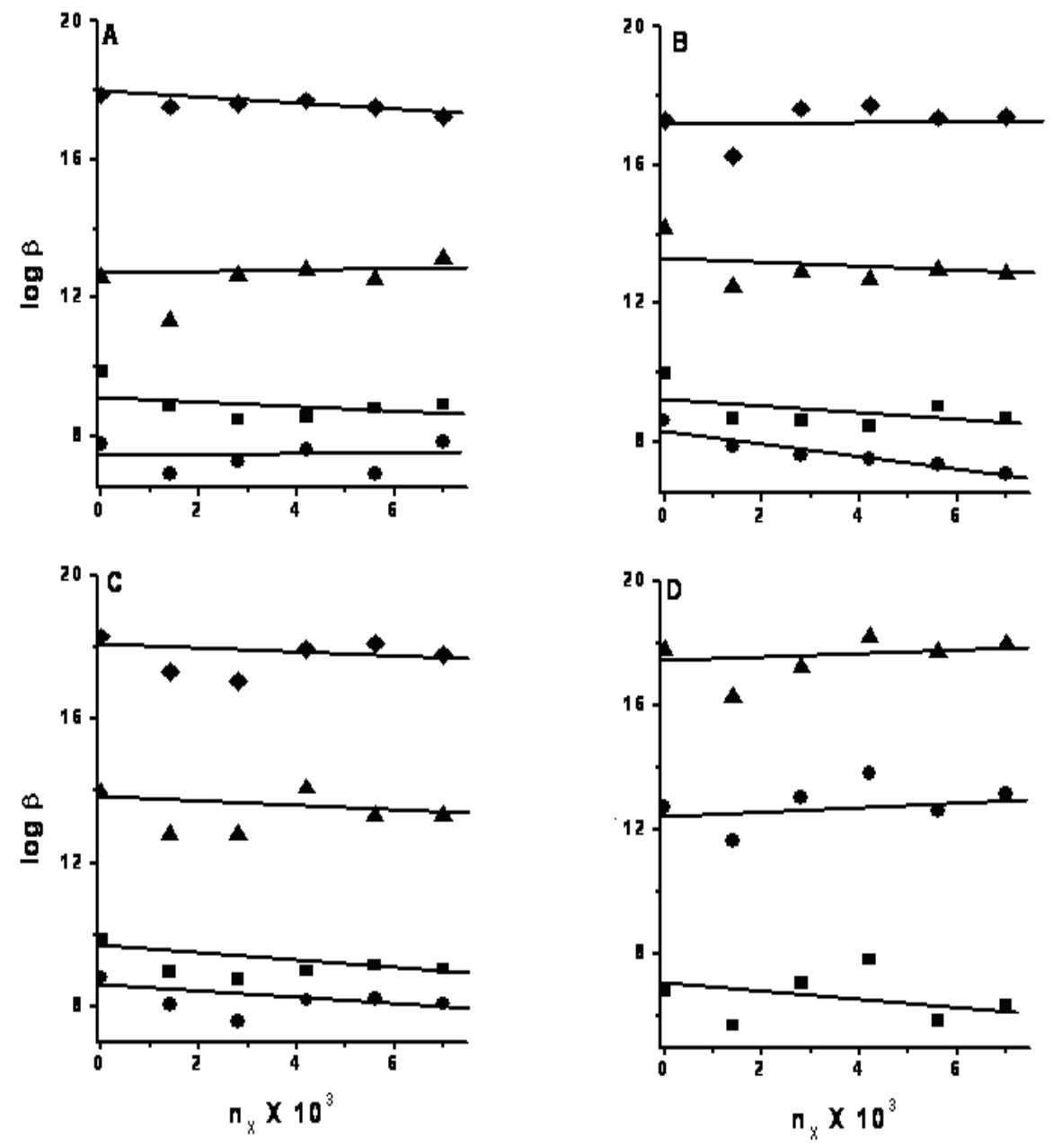

Figure 2. Variation of stability constant values of metal-citric acid complexes with mole fraction

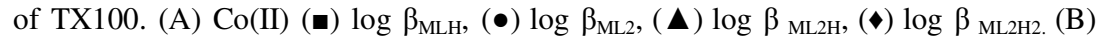
$\mathrm{Ni}(\mathrm{II})(\boldsymbol{\bullet}) \log \beta_{\mathrm{MLH}},(\bullet) \log \beta_{\mathrm{ML} 2},(\boldsymbol{\Delta}) \log \beta_{\mathrm{ML} 2 \mathrm{H}},(\bullet) \log \beta_{\mathrm{ML} 2 \mathrm{H} 2}$. (C) $\mathrm{Cu}$ (II) (๘) $\log \beta_{\mathrm{MLH}}$, $(\bullet) \log \beta_{\mathrm{ML} 2},(\boldsymbol{\Delta}) \log \beta_{\mathrm{ML} 2 \mathrm{H}},(\bullet) \log \beta_{\mathrm{ML} 2 \mathrm{H} 2}$. (D) $\mathrm{Zn}(\mathrm{II})(\boldsymbol{\bullet}) \log \beta_{\mathrm{ML} 2},(\bullet) \log \beta_{\mathrm{ML} 2 \mathrm{H}},(\boldsymbol{\Delta})$ $\log \beta_{\mathrm{ML} 2 \mathrm{H} 2 \text {. }}$ 
Micellar effect on metal-ligand complexes of $\mathrm{Co}(\mathrm{II}), \mathrm{Ni}(\mathrm{II}), \mathrm{Cu}(\mathrm{II})$ and $\mathrm{Zn}$ (II) with citric acid 355
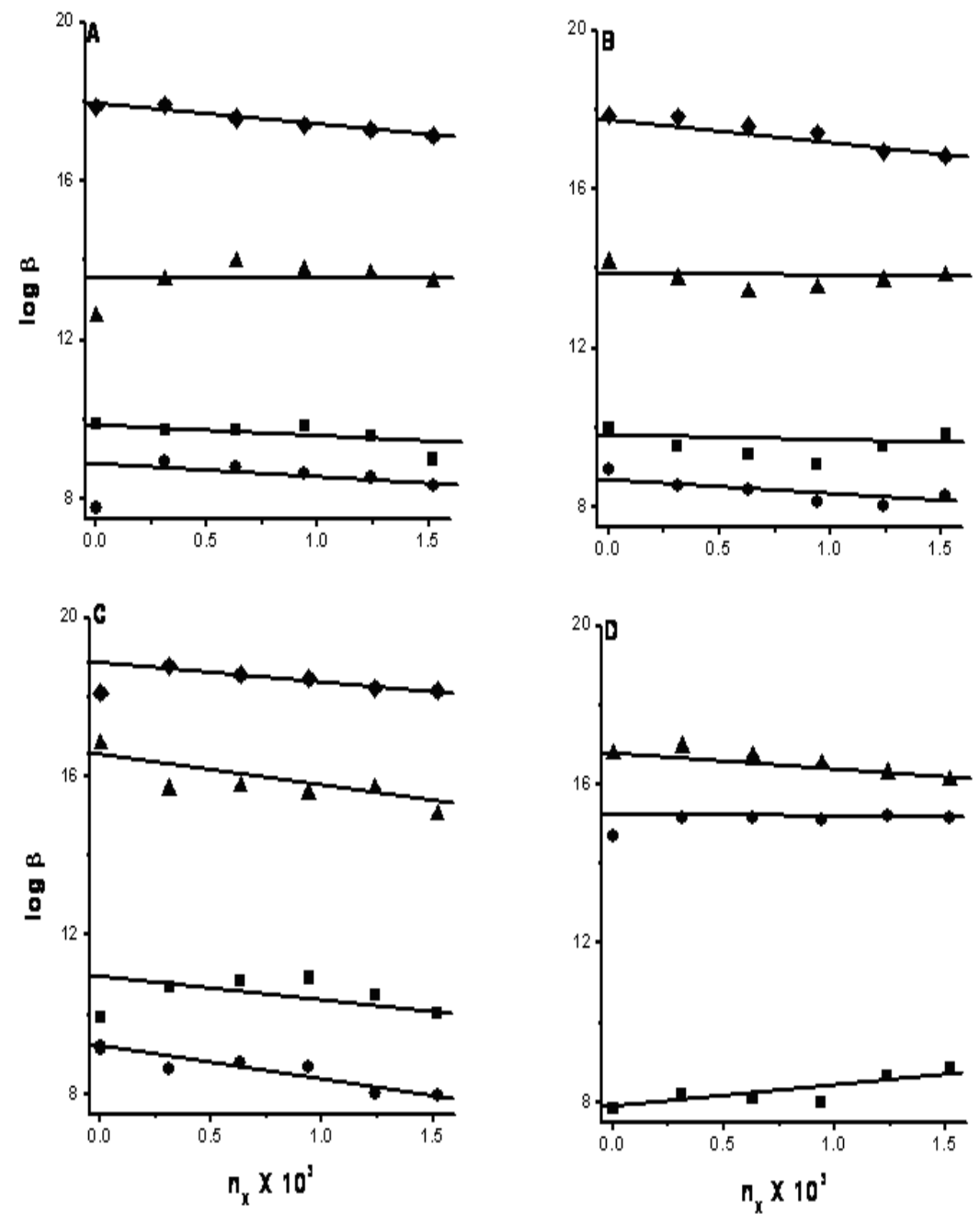

Figure 3. Variation of stability constant values of metal-citric acid complexes with mole fraction

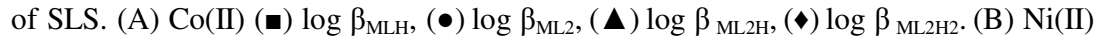
(•) $\log \beta_{\mathrm{MLH}},(\bullet) \log \beta_{\mathrm{ML} 2},(\boldsymbol{\Delta}) \log \beta_{\mathrm{ML} 2 \mathrm{H}},(\bullet) \log \beta_{\mathrm{ML} 2 \mathrm{H} 2}$. (C) $\mathrm{Cu}(\mathrm{II})(\boldsymbol{\bullet}) \log \beta_{\mathrm{MLH}},(\bullet)$ $\log \beta_{\mathrm{ML} 2},(\boldsymbol{\Delta}) \log \beta_{\mathrm{ML} 2 \mathrm{H}},(\bullet) \log \beta_{\mathrm{ML} 2 \mathrm{H} 2}$ (D) $\mathrm{Zn}(\mathrm{II})(\boldsymbol{\bullet}) \log \beta_{\mathrm{ML} 2},(\bullet) \log \beta_{\mathrm{ML} 2 \mathrm{H}},(\boldsymbol{\Delta}) \log$ $\beta_{\mathrm{ML} 2 \mathrm{H} 2}$.

The species $\mathrm{MLH}, \mathrm{ML}_{2}, \mathrm{ML}_{2} \mathrm{H}$ and $\mathrm{ML}_{2} \mathrm{H}_{2}$ exist for $\mathrm{Co}(\mathrm{II}), \mathrm{Ni}(\mathrm{II}), \mathrm{Cu}(\mathrm{II})$ and $\mathrm{ML}_{2}, \mathrm{ML}_{2} \mathrm{H}$ and $\mathrm{ML}_{2} \mathrm{H}_{2}$ exist for $\mathrm{Zn}(\mathrm{II})$. The above species exist in the $\mathrm{pH}$ range 1.6-7.5. The major chemical species, $\mathrm{ML}_{2} \mathrm{H}$ and $\mathrm{ML}_{2}$ exist to an extent of $60-70 \%$ for all the metal ions. The $\mathrm{ML}_{2} \mathrm{H}_{2}$ species is minor for all the four metals. The percentage of MLH species is the highest for $\mathrm{Ni}(\mathrm{II})$ system. 
Table 5. Best fit chemical models of acid-base equilibria of citric acid in SLS, CTAB and TX 100-water mixtures; temp $=303 \mathrm{~K}$, ionic strength $=0.16 \mathrm{M}$

\begin{tabular}{|c|c|c|c|c|c|c|c|c|c|c|}
\hline $\begin{array}{c}\% \\
\text { Surfactant }\end{array}$ & 011 & $\begin{array}{c}\log \\
\beta_{\mathrm{mlh}}(\mathrm{SD}) \\
012 \\
\end{array}$ & 013 & NP & $\mathrm{U}_{\text {corr }}$ & $\begin{array}{l}\text { Skew- } \\
\text { ness }\end{array}$ & $\chi^{2}$ & R-factor & Kurtosis & $\mathrm{pH}$ range \\
\hline \multicolumn{11}{|c|}{ SLS } \\
\hline 0.0 & $5.69(1)$ & $10.07(2)$ & 12.971) & 114 & 0.076 & -0.80 & 13.68 & 0.0073 & 4.98 & $2.0-6.8$ \\
\hline 0.5 & $5.92(1)$ & $10.46(1)$ & $13.53(2)$ & 107 & 0.371 & 0.12 & 18.67 & 0.0086 & 4.40 & $2.0-6.2$ \\
\hline 1.0 & $5.90(1)$ & $10.38(1)$ & $13.40(2)$ & 140 & 3.607 & -1.16 & 42.51 & 0.0050 & 5.75 & $1.7-6.0$ \\
\hline 1.5 & $6.20(1)$ & $10.65(2)$ & $13.68(3)$ & 100 & 1.569 & -1.80 & 33.28 & 0.0224 & 11.35 & $2.2-8.0$ \\
\hline 2.0 & $5.78(2)$ & $10.11(2)$ & $13.21(3)$ & 96 & 0.775 & -1.56 & 10.83 & 0.0145 & 11.79 & $2.2-8.0$ \\
\hline 2.5 & $5.45(1)$ & $9.53(1)$ & $12.23(1)$ & 73 & 2.331 & -0.33 & 35.72 & 0.0226 & 6.00 & $2.5-6.5$ \\
\hline \multicolumn{11}{|c|}{ CTAB } \\
\hline 0.5 & $5.35(1)$ & $9.51(1)$ & $12.26(1)$ & 100 & 2.175 & -0.66 & 31.16 & 0.0065 & 7.45 & $2.0-5.5$ \\
\hline 1.0 & $5.95(1)$ & $10.52(1)$ & $13.70(1)$ & 103 & 4.801 & 0.65 & 7.38 & 0.0034 & 5.10 & $2.0-6.0$ \\
\hline 1.5 & $5.80(1)$ & $10.18(1)$ & $13.13(1)$ & 124 & 6.661 & -1.31 & 37.87 & 0.0038 & 10.03 & $1.8-6.5$ \\
\hline 2.0 & 5.81(1) & $10.23(1)$ & $13.23(1)$ & 130 & 6.763 & 0.58 & 75.75 & 0.0058 & 7.10 & $1.8-6.5$ \\
\hline 2.5 & $5.51(1)$ & $9.79(1)$ & $12.60(1)$ & 130 & 1.535 & 0.60 & 11.75 & 0.0056 & 6.06 & $1.9-6.2$ \\
\hline \multicolumn{11}{|c|}{ TX100 } \\
\hline 0.5 & $5.61(1)$ & $9.95(1)$ & $12.81(1)$ & 128 & 1.67 & 0.71 & 15.50 & 0.0040 & 7.64 & $1-9-6.2$ \\
\hline 1.0 & $5.68(1)$ & $10.09(1)$ & 12.97(1) & 103 & 3.49 & 0.06 & 20.80 & 0.0094 & 5.34 & $2.5-6.0$ \\
\hline 1.5 & $5.68(1)$ & $10.08(1)$ & 13.11(1) & 129 & 1.79 & 0.13 & 48.48 & 0.0067 & 12.0 & $1.9-7.0$ \\
\hline 2.0 & $5.79(1)$ & $10.22(1)$ & $13.29(1)$ & 128 & 1.94 & 0.75 & 49.13 & 0.0064 & 10.3 & 1.9-7.0 \\
\hline 2.5 & $5.66(1)$ & $10.06(1)$ & $13.05(1)$ & 162 & 1.54 & 0.10 & 25.78 & 0.0046 & 4.71 & $1.6-7.0$ \\
\hline
\end{tabular}

Distribution diagrams were drawn using the formation constants of the best fit models and are shown in Figure 4. The pattern of the distribution of species with $\mathrm{pH}$ shows that the concentrations of species were affected by surfactant-water mixtures. The formation equilibria, based on the above observations, are represented below.

$$
\begin{aligned}
& \mathrm{M}(\mathrm{II})+\mathrm{LH}_{3} \rightleftharpoons \mathrm{MLH}_{2}^{+}+\mathrm{H}^{+} \\
& \mathrm{MLH}_{2}^{+} \rightleftharpoons \mathrm{MLH}+\mathrm{H}^{+} \\
& \mathrm{MLH} \rightleftharpoons \mathrm{ML}^{-}+\mathrm{H}^{+} \\
& \mathrm{MLH}_{2}^{+}+\mathrm{LH}_{3} \rightleftharpoons \mathrm{ML}_{2} \mathrm{H}_{4}+\mathrm{H}^{+} \\
& \mathrm{ML}_{2} \mathrm{H}_{4} \rightleftharpoons \mathrm{ML}_{2} \mathrm{H}_{3}^{-}+\mathrm{H}^{+} \\
& \mathrm{MLH}^{2} \mathrm{LH}_{3} \rightleftharpoons \mathrm{ML}_{2} \mathrm{H}_{3}^{-}+\mathrm{H}^{+} \\
& \mathrm{ML}_{2} \mathrm{H}_{3}{ }^{-} \rightleftharpoons \mathrm{ML}_{2} \mathrm{H}_{2}^{2-}+\mathrm{H}^{+} \\
& \mathrm{ML}_{2} \mathrm{H}_{2}^{2-} \rightleftharpoons \mathrm{ML}_{2} \mathrm{H}^{3-}+\mathrm{H}^{+} \\
& \mathrm{ML}_{2} \mathrm{H}^{3-} \rightleftharpoons \mathrm{ML}_{2}^{4-}+\mathrm{H}^{+}
\end{aligned}
$$

Some of the species shown in the above equilibria are not detected may be because the process is minor or the species is unstable. 
The cationic micelles (CTAB) have positive surface charge which can easily form complexes with negatively charged species. Hence, the stabilities of the complexes are increasing linearly with mole fraction of CTAB. The equilibria (b), (h) and (i) are more relevant in this context. TX100 is neutral surfactant. Hence, the equilibria involving decreasing charge on the complex species are favored by this surfactant. The equilibrium (b) is appropriate in this regard because a neutral species (MLH) is formed from a charged species $\left(\mathrm{MLH}_{2}{ }^{+}\right)$. The anionic micelles (SLS) have negatively charged surfaces which stabilize cationic complexes.
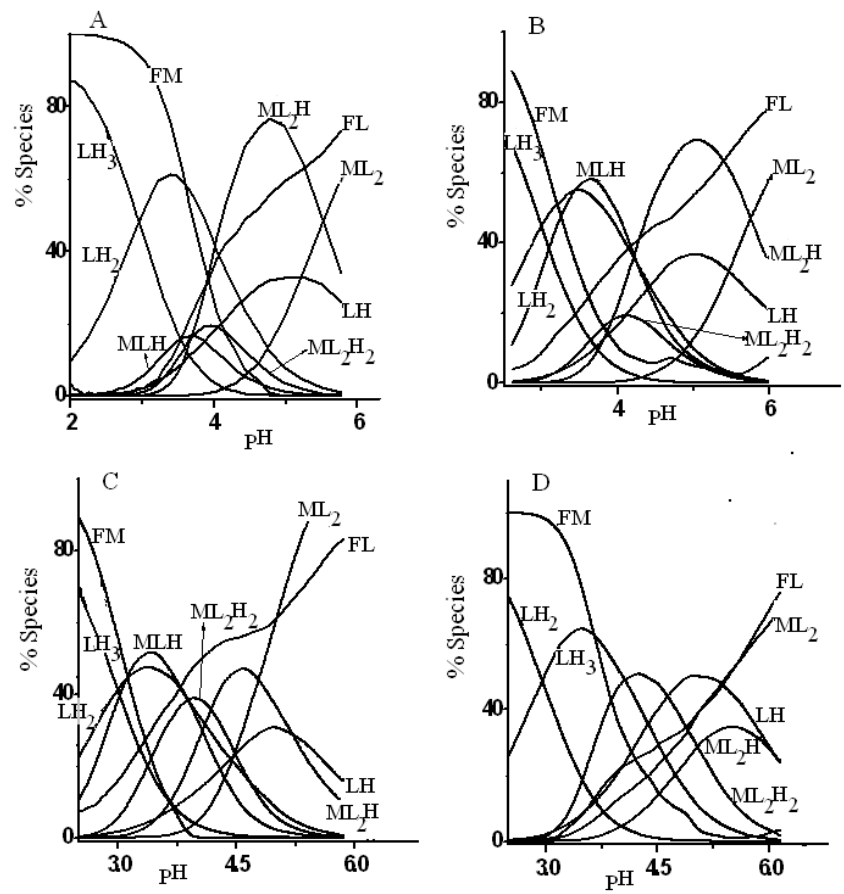

Figure 4. Distribution diagrams of citric acid complexes in $1.0 \% \mathrm{w} / \mathrm{v}$ SLS-water mixture. (A) $\mathrm{Co}$ (II), (B) $\mathrm{Ni}$ (II), (C) $\mathrm{Cu}$ (II) (D) and $\mathrm{Zn}$ (II).

\section{CONCLUSIONS}

On the basis of this study the following conclusions have been drawn. (1) The biomimic studies of metal ion complexes with citric acid indicate that all the complexes are protonated under acidic pH. (2) The linear variation of $\log \beta$ value of complexes with mole fraction of the surfactant indicates that electrostatic forces dominate the non-electrostatic forces. (3) Co(II), $\mathrm{Ni}(\mathrm{II}), \mathrm{Cu}(\mathrm{II})$ and $\mathrm{Zn}$ (II) interacted with citric acid in micellar media (CTAB, SLS and TX100) to form $\mathrm{ML}_{2}, \mathrm{ML}_{2} \mathrm{H}, \mathrm{ML}_{2} \mathrm{H}_{2}$ binary species. All the metals except zinc formed MLH species.

\section{REFERENCES}

1. Underwood, E.J. Trace Elements in Human and Animal Nutrition, 4th ed., Academic Press: New York; 1977; p 1. 
2. Poellot, R.A.; Shuler, T.R.; Uthus, E.O.; Nielsen, F.H. Proc. Natl. Acad. Sci. USA 1990, 44, 80 .

3. Lin, Y.L.; Lim C.J. J. Am. Chem. Soc. 2004, 126, 2602.

4. Cox, E.H.; Mc Lendon G.L. Curr. Opin. Chem. Biol. 2000, 4, 162.

5. Laity, J.H.; Lee, B.M.J.; Wright, P.E. Curr. Opin. Struct. Biol. 2001, 11, 39.

6. Dudev, T.; Lim, C.J. J. Chem. Soc. 2003, 50, 1093.

7. Ulrich, R. Organic Acids, in the Biochemistry of Fruits and Their Products, Vol. 1., Hulme, A.C. (Ed.), Academic Press: New York; 1970; p 89.

8. Portman, O.W.; Harris, R.S.; Nutritional requirements of nonhuman primates in Feeding and Nutrition of Nonhuman Primates, Academic Press: New York; 1970; 117.

9. Bozena, Z.; Iwona, O.; Arch. Immunol. Ther. Exp. 2006, 54, 69.

10. Guiwen, C.W.; Carlos, C.H. J. Am. Soc. Hort. 1997, 122, 95.

11. Masui, T.; Hirai, H.; Manaka, N.I.; Adachic G. J. Mat. Sci. Lett. 2002, 21, 489.

12. Richai-Noori, N.; Sarral-Mamoory, R. J. Cermic Processing Research 2008, 9, 246.

13. Young Jeong, I.; Seong-Taek, Kim.; Shu-Guang, Jin.; Hyang-Hwa, Ryu.; Yong-Hao, Jin.; Tae-Young Jung.; In-Young Kim.; Shin Jung. J. Pharm. Sci. 2007, 97, 1268.

14. Mukherjee, P.; J. Phys. Chem. 1962, 66, 943.

15. James, A.D.; Robinson, B.H. J. Chem. Soc. Faraday I 1978, 74, 10.

16. Mukerjee, P.; Mysels, K.J. Critical Micellar Concentrations of Aqueous Surfactant Systems, National Bureau of Standards: Washington; 1971.

17. Hinze, W.L. Solution Chemistry of Surfactants, Vol. 1, Plenum Press: NewYork; 1979.

18. Lavanya, K.V.; Rao, V.M.; Rao, G.N. Oxidat. Commun. 2008, 31, 398.

19. Latha, M.P.; Rao, V.M.; Rao, T.S.; Rao, G.N. Bull. Chem. Soc. Ethiop. 2007, 21, 63.

20. Rao, G.N.; Sudarsan, K.G. Chem. Spec. Bioavail. 2006, 18, 71.

21. Rao, G.N.; Ramakrishna, A. Proc. Natl. Acad. Sci. India 2005, 4, 75.

22. Gran, G. Analyst 1952, 77, 661.

23. Gran, G. Anal. Chim. Acta 1988, 206, 111.

24. Sailaja, B.B.V.; Kebede, T.; Rao, G.N.; Rao, M.S. P. Proc. Natl. Acad. Sci. (India) 2004, 74, 399.

25. Jeffery, G.H.; Bassett, J.; Mendham, J.; Denney, R.C. (Eds.), Vogel's Text Book of Quantitative Chemical Analysis, 5 th ed., Longmain: London; 1991; p 557.

26. Padmaja, N.; Babu, M.S.; Rao, G.N.; Rao, R.S.; Ramana, K.V. Polyhedron 1990, 9, 2497.

27. Rao, G.N. Ph.D. Thesis, Andhra University, Visakhapatnam, India, 1989.

28. Braibanti, A.; Rao, R.S.; Babu, A.R.; Rao, G.N. Ann. Chim. (Italy) 1995, 85, 17.

29. Gans, P.; Sabatini, A.; Vacca, A. Inorg. Chim. Acta 1976, 18, 237.

30. Bunton, C.A.; Romsted, L.S.; Supulveda, L. J. Phys. Chem. 1980, 84, 2611.

31. Chaimovich, H.; Politi, M.J.; Bonilha, J.B.S.; Quina, F.H. J. Phys. Chem. 1979, 83, 1951.

32. Born, M. Z. Phys. 1920, 1, 45.

33. Bunton, C.A.; Cerichelli, G.; Ihara Y.; Supulveda, L. J. Am. Chem. Soc. 1979, 101, 2429.

34. Rao, P.S.; Srikanth, B.; Rao, V.S.; Sastry, C.K.; Rao, G.N. E- J. Chem. 2009, 6, 561.

35. Srikanth, B.; Rao, P.S.; Rao, V.S.; Sastry C.K.; Rao, G.N.; Proc. Natl. Acad. Sci. India; 2009, 79, 335. 